\title{
Measuring Telomere Length as It Relates to Idiopathic Pulmonary Fibrosis Using a Novel Semi-Automated Approach to Imaging and Quantitation
}

\author{
Jonathan Boyd, Annik Migneault and Raphael Lemaire \\ AstraZeneca, Gaithersburg, Maryland, United States
}

Cellular senescence in type 2 alveolar epithelial cells type (AECII) is a hallmark of idiopathic pulmonary fibrosis (IPF). AECII senescence in IPF is due to a pathological and accelerated replicative telomere shortening. Therefore, developing a methodology to accurately and consistently quantify telomeres is critical for the experimental determination of the mechanisms underlying the onset of senescence. To build the pipeline required for measuring telomere length we compared telomere length in wild type (WT), 1218 weeks old female mice (C57BL-6) to telomerase reverse transcriptase knockout (KO) mice that display shortened telomeres. Lung sections were prepared from paraffin embedded tissue. Following deparaffinization, tissue was stained with DAPI to mark nuclei and an antibody against surfactant protein C (SPC), followed by secondary antibody conjugated with AF647, as a marker for AECII cells. To detect telomeres, in situ hybridization was performed using a Cy3-conjugated peptide nucleic acid (PNA) probe specific to telomere sequence. Accurate representation of telomere length throughout the lung required imaging a large area at super resolution. We used the Zeiss 880 Airy Scanner confocal microscope in super resolution mode, using a 63x 1.4 NA Oil Plan Apo objective, to image AECII cells over two hundred contiguous fields covering an area larger than $6 \times 6 \mathrm{~mm}$. These images were streamed to the processing workstation for Airy scan processing, often resulting in file sizes up to one terabyte. We collaborated with Arivis to develop software packages capable of handling, processing and quantifying data contained within these images. A watchdog module from Arivis 4D software identifies the Zeiss processed image on the image workstation and converts it into Arivis 4D .sis format. The images are then automatically moved to analysis workstation capable of analyzing images in a batched manner. The Arivis 4D software used a low and a high-resolution analysis pipeline to find the AECII cells and measure the length of the telomeres within the nuclei of each cell. The data is exported in a CSV file format that is easily read and graphed in Microsoft Excel. Using the WT and KO quantitation as benchmarks to determine the effect of different drugs on repairing telomere shortening, we successfully demonstrate the development of a semiautomated pipeline for the measurement of telomere length. This pipeline will enable confident analysis of novel drug targets aimed at reversing or preventing the onset of IPF related to AECII replicative senescence.

\section{References}

Elife. 2018 Jan 30;7. pii: e31299. doi: 10.7554/eLife.31299.

Therapeutic effects of telomerase in mice with pulmonary fibrosis induced by damage to the lungs and short telomeres.

Povedano JM\#1, Martinez P\#1, Serrano R1, Tejera Á1, Gómez-López G2, Bobadilla M3,4, Flores JM5, Bosch F6, Blasco MA1 